\title{
Recommended action plans for oral health in older Europeans
}

Anastassia E. KOSSIONl

DDS, MSc, PhD

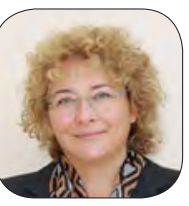

The current oral health delivery system fails to meet the oral health care needs of many population groups, including the frail and care-dependent older people.

Physical and mental illness, frailty and care dependency, socioeconomic inequalities, living in nursing homes, unhealthy diet, smoking, poor oral health literacy, lack of effective oral health policies including limited public dental care coverage, and limited training of healthcare professionals in oral conditions are some of the barriers to oral health faced by older persons [1-3]. Frail older people rarely visit the dentist, but they frequently meet other healthcare providers, such as physicians, nurses, physical therapists, occupational therapists, and pharmacists. However, few of those healthcare providers are adequately trained to initially assess oral problems and offer oral hygiene advice, as, for many years general and oral health have been viewed in isolation [3]. Consequently, oral health conditions in older people often remain underdiagnosed and untreated, leading to high prevalence of tooth loss, dental decay, periodontal disease, xerostomia, denture-related conditions and oral pre-cancer and cancer $[4,5]$. Moreover, there is evidence of an association between oral and general disease, such as cardiovascular disease, diabetes mellitus, respiratory disease and frailty [4,6-8].

In response to these challenges, the European College of Gerodontology (ECG) and the European Geriatric Medicine Society (EuGMS) have developed policy recommendations on oral health in older Europeans [9]. This expert opinion document identified three major areas for further action: a) educational plans for healthcare professionals, b) health policy plans, and c) citizens' empowerment and involvement.

The authors highlighted the importance of training non-dental healthcare professionals in oral health assessment and promotion, and provided a list of learning objectives as a basis for a dedicated "geriatric oral health curriculum" [9]. Healthcare professionals should be competent to initially assess oral health, identify oral conditions, discriminate between normal and abnormal findings in the mouth, decide when to refer to the dentist, demonstrate oral hygiene measures to older people and their caregivers, assist in daily oral hygiene provision, and work collaboratively with the elders' healthcare team. Moreover, although more than 86\% of the European dental schools teach Gerodontology at the undergraduate level, more dental training is necessary in oral health assessment and provision in nursing homes and at home, and in collaborative practice between dentists and other healthcare professionals [10].

The ECG/EuGMS recommendations further provided a list of health policy measures with emphasis on the integration of oral health into general health care assessment, prevention, provision and funding, and development of appropriate policies and protocols for oral health prevention and promotion in institutional settings for older residents [9]. Minimum requirements for nursing homes should include oral health assessment at entry, access to emergency and routine dental care, regular training of caregivers in oral hygiene provision to residents, provision of daily dental and denture hygiene and provision of regular oral screenings $[9,11,12]$. Other suggested actions included removing barriers to dental care, promoting domiciliary care, and promoting 
geriatric oral health campaigns for the public.

Finally, the document stressed the need for direct citizen involvement in actions related to oral health. Well informed citizens may guide decision-makers in enacting legislation for oral health promotion in frail older people and coverage by state or social security.

Given that the major chronic oral conditions, such as caries, periodontal disease, tooth loss, and many oral lesions are largely preventable and share common risk factors with chronic general diseases [4], it is imperative for healthcare, welfare, and educational authorities, public health planners and citizen organizations, to support the above ECG/EuGMS recommendations aiming to improve oral health and quality of life in older people.

\section{Acknowledgements}

The author would like to thank the co-authors of the ECG/EuGMS expert opinion document (in alphabetical order): Dr Justyna Hajto-Bryk, Professor Stefania Maggi, Dr Gerry McKenna, Professor Frauke Müller, Professor Mirko Petrovic, Professor Regina Elisabeth Roller-Wirnsberger, Professor Martin Schimmel, Professor Marija Tamulaitienè, and Professor Jacques Vanobbergen.

\section{Conflict of interest}

The author declares no conflict of interest with any financial organization regarding the material discussed in this document.

\section{AE Kossioni}

Associate Professor, Division of Gerodontology, Department of Prosthodontics, School of Dentistry, National and Kapodistrian University of Athens, Greece Leader of the Task \& Finish Group on Gerodontology by the European College of Gerodontology and the European Geriatric Medicine Society

References

1. Strayer MS. Perceived barriers to oral health care among the homebound. Spec Care Dent. 1995;15(3):113-118.

[Full text links] [PubMed] Google Scholar (41) Scopus (21)

2. Borreani E, Wright D, Scambler S, Gallagher]E. Minimising barriers to dental care in older people. BMC Oral Health. 2008; 8:7. doi: 10.1186/14726831-8-7.

[Full text links] [Free PMC Article] [PubMed] Google Scholar (53) Scopus (34)

3. Kossioni AE. ls Europe prepared to meet the oral health needs of older people? Gerodontology. 2012;29(2): e1230-1240. doi: 10.1111/j.17412358.2010.00445.x.

[Full text links] [PubMed] Google Scholar (17)

4. Petersen PE, Yamamoto T. Improving the oral health of older people: the approach of the WHO Global Oral Health Programme. Community Dent Oral Epidemiol. 2005;33(2):81-92. doi: 10.1111/j.1600-0528.2004.00219.x. [Full text links] [PubMed] Google Scholar (919) Scopus (395)

5. Kossioni AE. Current status and trends in oral health in the communitydwelling older adults- A global perspective. Oral Health Prev Dent. 2013;11(4):331-340. doi:10.329o/j.ohpd.azo171.

[Full text links] [PubMed] Google Scholar (13) Scopus (7)

6. Aida], Kondo K, Yamamoto T, et al. Oral health and cancer, cardiovascular, and respiratory mortality of Japanese.J Dent Res. 2011;90(9):1129-1135. doi: 10.1177/oo22034511414423. [Full text links] [PubMed] Google Scholar (42) Scopus (21)

7. Pace CC, McCullough GH. The association between oral microorganisms and aspiration pneumonia in the institutionalized elderly: review and recommendations. Dysphagia 2010; 25(4): 307-322. doi: 10.1007/soo455o10-9298-9. Review.

[Full text links] [PubMed] Google Scholar (152) Scopus(70)

8. Ramsay SE, Papachristou E, Watt RG, et al. Influence of poor oral health on physical frailty: a population-based cohort study of older British men. J Am Geriatr Soc. 2017:1-7. doi:10.1111/jgs.15175. [Full text links] [PubMed] Google Scholar (1)

9. Kossioni AE, Hajto-Bryk], Maggi S, et al. An expert opinion from the European College of Gerodontology and the European Geriatric Medicine Society: European policy recommendations on oral health in older adults. J Am Geriatr Soc. 2018;66:609-613. doi:10.111/jgs.15191.

[Full text links] [PubMed] Google Scholar (1)

10. Kossioni A, McKenna G, Müller F, Schimmel M, Vanobbergen J. Higher education in Gerodontology in European Universities. BMC Oral Health. 2017;17(1):71. doi: 10.1186/s12903-017-0362-9

[Full text links] [Free PMC Article] [PubMed] Google Scholar (2) Scopus (2)

11. Gil-Montoya A, de Mello AL, Cardenas CB, Lopez IG. Oral health protocol for the dependent institutionalized elderly. Geriatr Nurs. 2006;27(2):95-101. doi: 10.1016/j.gerinurse.2005.12.003 [Full text links] [PubMed] Google Scholar (81) Scopus (36)

12. Janssens B, De Visschere L, van der Putten G] et al. Effect of an oral healthcare protocol in nursing homes on care staffs' knowledge and attitude towards oral health care: A cluster-randomised controlled trial. Gerodontology. 2016;33(2):275-286. doi:10.1111/ger.12164 [Full text links] [PubMed] Google Scholar (8) Scopus (4) 